Check for updates

1 Johns Hopkins University School of Medicine, Department of Pediatrics, Division of Infectious Diseases, Baltimore, MD, USA

2 Johns Hopkins University School of Medicine, Department of Pediatrics, Division of General Pediatrics,

Baltimore, MD, USA

Correspondence to A Sick-Samuels asick1@jhmi.edu

Cite this as: BMJ 2021;372:n385 http://dx.doi.org/10.1136/bmj.n385 Published: 01 March 2021

\title{
CLINICAL UPDATES
}

\section{Acute covid-19 and multisystem inflammatory syndrome in children}

\section{Jessica H Rubens, ${ }^{1}$ Nadine Peart Akindele, ${ }^{1}$ Megan M Tschudy, ${ }^{2}$ Anna C Sick-Samuels ${ }^{1}$}

\section{What you need to know}

- Children with acute covid-19 can present with non-specific symptoms. Exclude covid-19 in children with fever and respiratory tract symptoms or loss of taste or smell, especially if there is possible exposure to others with the virus

- Consider multisystem inflammatory syndrome (MIS-C) in children presenting with fever and abdominal symptoms-particularly if they develop conjunctivitis or rash-and refer to a paediatric emergency department for evaluation

- MIS-C can have overlapping symptomatology with disease processes that require prompt treatment, such as sepsis, toxic shock syndrome, myocarditis, and meningitis. Therefore, consider initiation of empiric antibiotics and necessary evaluations if the patient develops cardiovascular or respiratory compromise, evidence of acute abdomen, or meningismus

- Simple prevention measures, including mask wearing, hand hygiene, and social distancing remain crucial to prevent the spread of SARS-CoV-2 in children and adults

\section{Presentation of SARS-CoV-2 infection in children}

The most common symptoms in children with acute SARS-CoV-2 infection (covid-19 disease) are fever and cough. Other symptoms may include sore throat, rhinorrhoea, or congestion, myalgias, headache, fatigue, and gastrointestinal symptoms including nausea, vomiting, or diarrhoea. The range of symptom prevalence is reported from three large meta-analyses, one with 131 studies and 7780 paediatric patients, ${ }^{1}$ the second with 28 studies and 1614 patients, $^{2}$ and the third with 46 studies and 551 patients ${ }^{3}$ and summarised in table 1 . Though children have a similar distribution of initial symptoms as compared with adults, children are more likely to have mild, self-resolving symptoms without progression to the lower pulmonary disease that necessitates hospitalisation. ${ }^{4}$ Dermatological manifestations in children with mild disease are uncommon: acute infection has at times been associated with a maculopapular exanthem, but the pseudo chilblain lesions or "covid toes" seen in adults are rare. ${ }^{56}$ Although assessing the prevalence of loss of taste or smell among children may be challenging (especially when children are unable to report these symptoms), ${ }^{7}$ as with adults, some studies suggest the presence of loss of taste or smell may also be relatively specific for covid-19 among children. ${ }^{8}$

Table 1 | Prevalence of symptoms in children with acute SARS-CoV-2 infection, summarised from three meta-analyses

\begin{tabular}{ll} 
Symptom & Range $^{1-3 *}$ \\
\hline Asymptomatic & $16-19 \%$ \\
\hline Fever & $48-59 \%$ \\
\hline Cough & $39-56 \%$ \\
\hline Rhinorrhoea, nasal congestion & $7-20 \%$ \\
\hline Myalgia & $14-19 \%$ \\
\hline Sore throat & $14-18 \%$ \\
\hline Headache & $3-13 \%$ \\
\hline Tachypnoea, dyspnoea & $8-12 \%$ \\
\hline Diarrhoea & $7-10 \%$ \\
\hline Nausea, vomiting & $2-9 \%$ \\
\hline Abdominal pain & $6-7 \%$ \\
\hline Fatigue & $5-8 \%$ \\
\hline Rash & $<1 \%$ \\
\hline (Early studies did not specify loss of taste or smell because younger children are not able to report this symptom) \\
*Hoang et al included 131 studies with 7780 patients; Assaker et al included 28 studies with 1614 patients; Zhang et al included 46 studies with 551 patients
\end{tabular}

Data that further disaggregate paediatric symptoms by age are relatively limited, but among infants the most common symptoms of acute covid-19 similarly include fever, cough, and nasal congestion. Infants may also present with isolated fever, poor feeding, or fussiness. 9 $^{-12}$ 


\section{Asymptomatic and mild disease}

Multiple reports have described a mild illness trajectory and high rates of asymptomatic infection in children with acute covid-19. A retrospective case series of 2135 Chinese children with suspected or confirmed acute SARS-CoV-2 infection found that more than $90 \%$ of patients were asymptomatic or had mild to moderate illness. ${ }^{13}$ The true incidence of asymptomatic SARS-CoV-2 infection is difficult to confirm, as asymptomatic children may not be tested, however, meta-analyses estimate that between $16 \%$ and $19 \%$ of paediatric cases are asymptomatic. ${ }^{1-3}$ A recent prospective multicentre study of four French hospitals screened all admitted patients for SARS-CoV-2 infection by polymerase chain reaction testing and found that $45 \%$ of the 438 positive paediatric cases hospitalised were asymptomatic. ${ }^{14}$ In another study, investigators fit age structured mathematical models to epidemiological data from multiple countries and estimated that clinical symptoms only manifest in $21 \%$ of infected children. ${ }^{15}$ As of February 2021, the US Centers for Disease Control and Prevention (CDC) estimated that children age 0-4 years account for only $1.9 \%$ of covid-19 diagnoses, and those age 5-17 years account for $9.4 \%$ of covid-19 diagnoses in the US. ${ }^{16}$

\section{Severe covid-19 disease}

A small proportion of children go on to develop severe acute covid-19 disease and require hospitalisation because of respiratory compromise or complications of SARS-CoV-2 infection such as multisystem inflammatory syndrome in children (MIS-C). A CDC study in August 2020 of 576 children hospitalised with SARS-CoV-2 infection reported a lower hospitalisation rate compared with adults (8 versus 165 per 100 0oo); however, one in three hospitalised children required admission to intensive care units, a rate similar to adults. ${ }^{4}$ This report also describes a bimodal distribution in hospitalisation rate by age. Infants <2 months accounted for $19 \%$ of admissions-which may reflect a general practice standard to admit and evaluate neonates with fever or other symptoms that could indicate a serious bacterial infection rather than disease severity-because most neonates have mild self-resolving infections with brief admissions. ${ }^{17}$ Children age $12-17$ years accounted for $42 \%$ of admissions, which may reflect more severe illness in adolescents. ${ }^{4}$ It appears that children who become severely ill with acute covid-19 often have one or more underlying conditions, including medical complexity, obesity, asthma, sickle cell disease, and immunosuppression. ${ }^{418}$ Reassuringly, the mortality rate in paediatric patients remains low, accounting for $<1 \%$ of all deaths associated with SARS-CoV-2 in the US. ${ }^{118} 19$ The risk factors for severe illness in children will likely be further clarified by ongoing research.

\section{Community transmission of SARS-CoV-2 is happening in my region. How should I approach a sick child?}

In places where there is active community transmission of SARS-CoV-2, children with fever, respiratory tract symptoms, loss of taste or smell, or multiple infectious symptoms should undergo testing for covid-19 or be considered to have the disease until proved otherwise. Acute covid-19 does not appear to be easily distinguished from other viral infections based on symptomatology. A retrospective cohort study compared children infected with influenza $(n=1402)$ or SARS-CoV-2 $(n=315)$ and found that those admitted with SARS-CoV-2 reported more frequent non-specific viral symptoms than those with influenza, but no difference was seen in the rate of hospitalisation, intensive care unit admission, or mechanical ventilation. ${ }^{20}$ In addition, a systematic review suggested that $6 \%$ of children with covid-19 also had a concurrent bacterial or viral co-infection. ${ }^{1}$ Though children with fever and respiratory tract symptoms will likely undergo testing for SARS-CoV-2 where covid-19 is endemic, clinicians should consider a range of other diagnoses, including other pulmonary infections and systemic illnesses with respiratory manifestations, including non-infectious diagnoses such as diabetic ketoacidosis (table 2). Encourage parents or caregivers to return to care if their child develops symptoms that suggest more serious disease, including rapid or laboured breathing, dehydration, persistent fever, severe abdominal pain, or altered mental status.

Table 2 | Differential diagnosis of acute covid-19 and multisystem inflammatory disease (MIS-C)

\begin{tabular}{|c|c|}
\hline $\begin{array}{l}\text { Acute covid-19 } \\
\text { (chief concern: respiratory tract symptoms) }\end{array}$ & $\begin{array}{l}\text { MIS-C } \\
\text { (chief concern: fever with abdominal pain and/or rash) }\end{array}$ \\
\hline \multicolumn{2}{|l|}{ Infectious causes } \\
\hline $\begin{array}{l}\text { Other respiratory viral infections (eg, influenza, adenovirus, respiratory syncytial virus, rhinovirus) } \\
\text { causing upper and/or lower respiratory tract infection } \\
\text { Atypical pneumonia } \\
\text { Acute bacterial pneumonia } \\
\text { Bacterial sinusitis } \\
\text { Tuberculosis }\end{array}$ & $\begin{array}{l}\text { Acute covid-19 or other viral infections (eg, adenovirus, enterovirus/coxsackie viruses) } \\
\text { Toxic shock syndrome } \\
\text { Bacterial sepsis } \\
\text { Appendicitis } \\
\text { Abdominal abscess }\end{array}$ \\
\hline \multicolumn{2}{|l|}{ Non-infectious causes } \\
\hline $\begin{array}{l}\text { Reactive airway disease/asthma } \\
\text { Cardiac dysfunction } \\
\text { Hyperglycaemia } \\
\text { Dehydration } \\
\text { Diabetic ketoacidosis } \\
\text { Foreign body }\end{array}$ & $\begin{array}{l}\text { Intussusception } \\
\text { Myocarditis } \\
\text { Kawasaki disease } \\
\text { Haematological malignancy } \\
\text { Macrophage activation syndrome } \\
\text { Haemophagocytic lymphohistiocytosis } \\
\text { Systemic juvenile idiopathic arthritis } \\
\text { Systemic lupus erythematosus }\end{array}$ \\
\hline
\end{tabular}

Children with fever and gastrointestinal symptoms (abdominal pain, vomiting, or diarrhoea) or any child with other features consistent with Kawasaki disease (eg, persistent fever plus lymphadenopathy, mucocutaneous changes, conjunctivitis, or swelling of extremities) could have MIS-C. These patients require urgent evaluation in an emergency department, preferably staffed by paediatric specialists. 


\section{Multisystem inflammatory disease in children}

MIS-C (box 1) is thought to be a hyperinflammatory syndrome that develops approximately 2-6 weeks after SARS-CoV-2 infection.

\section{Box 1: Case definition of MIS-C}

- Presence of fever for $\geq 24$ hours

- Elevated inflammatory markers

- Multi-organ dysfunction ( $\geq 2$ systems: cardiac, dermatological, gastrointestinal, renal, respiratory, haematological, and/or neurological)

- No plausible alternative diagnosis

- Positive viral or serological testing for SARS-CoV-2 or close contact with a person with covid-19 within four weeks of symptom onset ${ }^{21}$

Typically, school aged children are affected (median age 8 years), though MIS-C has been reported in children from infancy through young adulthood. ${ }^{22-24}$ Children with MIS-C present with persistent fever, and most often have gastrointestinal symptoms (eg, abdominal pain, vomiting, diarrhoea), mucocutaneous changes (eg, rash, conjunctivitis), and may experience neurological symptoms (eg, headache, meningismus) (table 3). Patients with MIS-C may present with some features consistent with Kawasaki disease (particularly fever and mucocutaneous changes); however, MIS-C is likely a distinct clinical entity. Patients with MIS-C often have predominant cardiac dysfunction and gastrointestinal symptoms, in addition to biomarker and cytokine differences that appear distinct from Kawasaki disease. ${ }^{2326-28}$ One study observed that younger patients (aged 0-5 years) with MIS-C were more likely to meet Kawasaki disease or atypical Kawasaki disease criteria compared with adolescents ( $48 \% v 12 \%)$, had a higher prevalence of mucocutaneous symptoms $(87 \% v 62 \%)$, and had a lower prevalence of myocarditis $(39 \% v 73 \%)$ or neurological symptoms $\left(13 \% v 39 \%\right.$ of adolescents). ${ }^{26}$ Though there are hypotheses around risk factors for MIS-C, such as lower pre-existing immunity to coronaviruses, specific risk factors for developing MIS-C in children are not well established. ${ }^{28}$

Table 3 | Clinical features of patients with MIS-C, summarised from two surveillance studies and one meta-analysis

\begin{tabular}{lc} 
& Range $^{22-24 *}$ \\
Previously healthy & $66-73 \%$ \\
\hline Median age & 8 years \\
& (range 0-20 years) \\
\hline Organ system involvement & $87-92 \%$ \\
$\quad$ Gastrointestinal & $67-87 \%$ \\
Cardiovascular & $74-76 \%$ \\
Haematological & $71-74 \%$ \\
Mucocutaneous & $63-70 \%$ \\
Respiratory & $38-39 \%$ \\
\hline Neurological & $64-80 \%$ \\
\hline Admission to intensive care & $13-30 \%$ \\
\hline Mechanical ventilation & $42-48 \%$ \\
\hline Vasoactive support & $2-4 \%$ \\
\hline Death &
\end{tabular}

MIS-C is typically a progressive illness, and patients who initially had mild symptoms can develop severe illness with multi-organ dysfunction within a few days of symptom onset. Critical signs may include haemodynamic instability, tachycardia, left ventricular dysfunction, and respiratory distress, which could be primary or caused by cardiac dysfunction. Approximately two-thirds of patients in the US with MIS-C required admission to intensive care. ${ }^{23}$ and 30 deaths among 2060 patients with MIS-C have been reported in the US as of 8 February $2021 .{ }^{21}$ Laboratory abnormalities often include lymphopenia, anaemia, and thrombocytopenia, in addition to elevations in liver enzymes, creatinine, pro-brain natriuretic protein, troponin, and coagulation studies. If MIS-C is suspected, it is important to obtain laboratory tests to evaluate for evidence of inflammation, cardiac, and other organ dysfunction, and to obtain additional diagnostic imaging based on physical examination findings or laboratory results. All patients in whom there is a strong suspicion for MIS-C should have an echocardiogram to evaluate cardiac function and to look for evidence of coronary artery dilatation. The presenting signs and symptoms of MIS-C in children can overlap with other time-sensitive diagnoses, such as sepsis, toxic shock, myocarditis, appendicitis, or meningitis. Therefore, it is important to consider these diseases processes, pursue evaluation, and initiate antibiotic therapy if necessary (table 2). Overall, MIS-C is a rare complication of SARS-CoV-2. A May 2020 systematic review from 26 countries reported a MIS-C incidence of $0.14 \%$ among all children with SARS-CoV-2 infection, ${ }^{1}$ but this estimated incidence may be imprecise because of potential underestimation of overall SARS-CoV-2 infections in children. Although it is rare, MIS-C is a critical diagnosis to consider in any child with fever, evidence of inflammation, and organ dysfunction where covid-19 is endemic. ${ }^{29}$ Prompt treatment with intravenous immunoglobulin and steroids can reduce the inflammatory response and may improve outcomes.

\section{Transmission dynamics of SARS-CoV- 2 in children}

While questions remain regarding the susceptibility of children to covid-19 when exposed to the SARS-CoV-2 virus and their ability to transmit it to others, transmission of the virus from children to contacts in their households, camps, and schools ${ }^{30-32}$ has been clearly documented, particularly when face masks are not worn universally. ${ }^{33}$ For example, a 13 year old boy whose only symptom was nasal congestion transmitted the infection to a household of people in the US. ${ }^{32}$ In contrast, when universal masking and distancing guidelines were used, a prospective observational study followed 90 ooo students and staff in a North Carolina school system for nine weeks and found 773 community acquired SARS-CoV-2 
infections, with a very low rate of within school infections (32 cases total). ${ }^{34}$ In addition, a recent CDC case-control study in Mississippi found that close contacts with anyone outside the household and inconsistent mask use in school was associated with SARS-CoV-2 infection, but school or day care attendance itself was not associated SARS-CoV-2 infection when masks were used. ${ }^{35}$ Though further research is needed to clarify viral transmission dynamics specific to the unique immunobiology of children, they are able to transmit the virus to others and appropriate prevention measures are important in all settings. If a household or other close contact contracts SARS-CoV-2, the exposed child should therefore quarantine and stay away from school or other activities. Family members may consider physical separation and wearing masks around other exposed household members when in common areas to try to reduce potential for household transmission, though this may not always be feasible.

\section{Considerations for testing}

If a child has symptoms of a viral infection and SARS-CoV-2 is circulating in the community, testing for SARS-CoV-2 infection is warranted. Even if symptoms are mild, confirming SARS-CoV-2 infection informs isolation and contact tracing of positive cases. For the purposes of school attendance, attempts have been made to risk stratify children with symptoms regarding likelihood of SARS-CoV-2. ${ }^{36-38}$ However, manifestations in an individual can vary greatly, the virus can transmit among asymptomatic people, and co-infections are possible. The local prevalence of SARS-CoV-2 and the child's personal exposures should be taken into

consideration. If testing is not available, regardless of the actual cause of symptoms, it is safest to keep children at home until fevers resolve and symptoms improve, following regional public health guidance on when they may return to school. Generally, SARS-CoV-2 testing among children is considered as reliable as testing in adults, because at least one study has shown that children with mild to moderate SARS-CoV-2 infection have similar or even higher nasopharyngeal SARS-CoV-2 viral loads compared with adults. ${ }^{39}$ Antibody testing is generally not recommended unless a patient is being evaluated for MIS-C, as the presence or absence of antibodies would not otherwise inform clinical management or modify the need for preventive behaviours. 40

\section{Health disparities in clinical outcomes and social determinants of health}

The covid-19 pandemic has dramatically highlighted health disparities among racial and ethnic groups. In the US, children of Latinx, Black, and minority ethnicities experienced higher rates of hospitalisation owing to SARS-CoV-2 compared with non-Hispanic white children ( 16.4 V 10.5 per 100 00o), 4 and Indigenous Americans/Alaska Natives were 5.3 times more likely to be hospitalised..$^{41}$ In the UK, a prospective study found that Black children were over-represented in their cohort with increased odds of admission to intensive care. Children with MIS-C were also more likely to be of non-white ethnicity $(64 \% v 42 \%)$, which may reflect increased rate of covid-19 infection in their communities. ${ }^{42}$ These racial and ethnic differences may reflect vulnerabilities to viral transmission related to occupational exposures, housing arrangements, or need to use public transportation. These factors, in addition to limitations in healthcare access and systemic inequities, contribute to the disparities highlighted by the covid-19 pandemic. Moreover, school closures disproportionately affect children from low income communities by affecting in-person learning services, while also jeopardising nutrition, medical, and mental health services provided through schools. Given that ethnic minorities and low income families are also disproportionately more likely to experience unemployment, home foreclosures, evictions, and other financial insecurities as a result of the pandemic, the mental health impacts of the pandemic on these families and their children are likely to be vast and enduring. Consider asking families about financial and psychosocial stressors and connect families with local support services, where available.

\section{Therapeutic and prevention efforts}

Children with mild acute covid-19 benefit from usual supportive care measures, including rest, hydration, and antipyretics as needed. Dexamethasone was shown to decrease mortality in adults with moderate to severe respiratory distress, and may be considered in children with significant respiratory illness, though paediatric data are still forthcoming. ${ }^{43}$ Similarly, though efficacy is still being evaluated in children, remdesivir may be prescribed for children with respiratory deterioration. Other treatments, such as convalescent plasma or monoclonal antibodies, might be considered in high risk patients, but these therapeutics require further study in adults and children. Children with MIS-C most commonly are treated with intravenous immunoglobulin and often steroids. Data on outcomes and treatment efficacy is limited overall. However, a recent retrospective cohort study in France found that children with MIS-C who were treated with both intravenous immunoglobulin (IVIg) and methylprednisolone $(n=72)$ had a lower risk of treatment failure compared with those treated with IVIg alone $(n=34) .{ }^{29}$ Further study is still needed to understand the pathophysiology of MIS-C to inform the most effective therapeutic interventions.

Primary measures to prevent infection and transmission of SARS-CoV-2 remain important for children and their families and include basic steps such as face masks for children aged 2 years and older, social distancing, and hand hygiene for both children and the adults around them. Young children or those with developmental delays may not tolerate or wear masks properly; however, there is value in practising. Several vaccines have been authorised for emergency use in persons $\geq 16$ or 18 years. Given that the risks associated with SARS-CoV-2 are much lower in children than in adults, initial studies and vaccine distribution did not prioritise children. Fortunately, several studies are now enrolling younger children as it will be important to understand vaccine safety and efficacy before vaccines are widely administered to younger age groups. Patients should maintain routine preventive care and vaccination schedules, including seasonal influenza vaccine, as a critical strategy to stay healthy during and beyond the pandemic. Families should continue to follow infection prevention measures, even after individual vaccination, until community rates are low to avoid the potential for asymptomatic transmission to others.

\section{Uncertainties}

- Are children less susceptible to acquiring SARS-CoV-2 infection? If so, what are the mechanisms for decreased susceptibility?

- What is the pathophysiology of MIS-C? What are the risk factors for developing MIS-C?

- Why are some racial and ethnic minority populations at increased risk of severe SARS-CoV-2 associated illness and death? How can this risk be mitigated?

\section{Education into practice}

- If SARS-CoV-2 is circulating in your community and a child presents with fever, cough, or congestion, what differential diagnoses should you consider? 
- How might infants present differently from older children with SARS-CoV-2 infection?

- What are the most common presenting symptoms of MIS-C and what other diagnoses should also be considered?

\section{How this article was made}

We searched the Medline database and used a personal archive of references to prepare this update. We included articles that were peer reviewed, had rigorous study design, and had large sample sizes when possible. Articles focusing on paediatric age groups were prioritised. The primary literature review occurred in October 2020; however, we included key up-to-date publications through February 2021.

\section{How patients were involved in the creation of this article}

Patients (and their families) under the care of the authors were invited to be interviewed about their perspective on covid-19, as well as the effect of the pandemic on their family and their livelihood. Parents were confused regarding how their children had acquired the illness, particularly when SARS-CoV-2 antibodies were found during an evaluation for MIS-C. Some parents expressed concern about their own risk of acquiring SARS-CoV-2 while in a hospital setting with their sick child and were unsure of the possibility that they, as parents, may acquire the illness. Families recognised that there is still much to be learnt about the disease and its transmission-but were puzzled by the varied manifestations of infection in different people. Gaining a better understanding of the specific concerns and challenges facing families informed the focus of this article.

Competing interests: We have read and understood the BMJ policy on declaration of interests and declare the following interests: none.

Support: JHR is a fellow in the Paediatric Scientist Development Program; this work was supported by the Eunice Kennedy Shriver National Institute of Child Health and Human Development Award K12-HD000850 (JHR), and by the National Institutes of Health T32-AI052071 (JHR, NPA) and KL2TR003099 (ACS).

Contributors and guarantor: JR and AS conceptualised the article. JR conducted the initial literature review and prepared the first draft of the article. JR, NPA, MT, and AS contributed to additional literature review, revisions, and approved the final manuscript. AS is the guarantor.

Acknowledgements: We express gratitude to all the healthcare personnel caring for children and their families through this pandemic.

Provenance and peer review: commissioned; externally peer reviewed.

Hoang A, Chorath K, Moreira A, etal. COVID-19 in 7780 pediatric patients: A systematic review. EClinicalMedicine 2020;24:100433. doi: 10.1016/j.eclinm.2020.100433 pmid: 32766542

2 Assaker R, Colas AE, Julien-Marsollier F, etal. Presenting symptoms of COVID-19 in children: a meta-analysis of published studies. Br J Anaesth 2020;125:e330-2. doi: 10.1016/j.bja.2020.05.026 pmid: 32534738

3 Zhang L, Peres TG, Silva MVF, Camargos P. What we know so far about Coronavirus Disease 2019 in children: A meta-analysis of 551 laboratory-confirmed cases. Pediatr Pulmonol 2020;55:2115-27. doi: 10.1002/ppul.24869 pmid: 32519809

4 Kim L, Whitaker M, O'Halloran A, etalCOVID-NET Surveillance Team. Hospitalization rates and characteristics of children aged <18 years hospitalized with laboratory-confirmed COVID-19 -COVID-NET, 14 States, March 1-July 25, 2020. MMWR Morb Mortal Wkly Rep 2020;69:1081-8. doi: 10.15585/mmwr.mm6932e3 pmid: 32790664

5 Daneshgaran G, Dubin DP, Gould DJ. Cutaneous manifestations of COVID-19: an evidence-based review. Am J Clin Dermatol2020;21:627-39. doi: 10.1007/s40257-020-00558-4 pmid: 32865778

6 Gisondi P, Plaserico S, Bordin C, Alaibac M, Girolomoni G, Naldi L. Cutaneous manifestations of SARS-CoV-2 infection: a clinical update. J Eur Acad Dermatol Venereol 2020;34:2499-504. doi: 10.1111/jdv.16774. pmid: 32585074

7 Hall A, Frauenfelder C, Butler C, Coyle P, Hopkins C. Paediatric olfactory dysfunction: a chance to detect COVID-19?Arch Dis Child 2020;106:e17.

8 King JA, Whitten TA, Bakal JA, McAlister FA. Symptoms associated with a positive result for a swab for SARS-CoV-2 infection among children in Alberta. CMA/2021;193:E1-9.

9 Cui X, Zhao Z, Zhang T, etal. A systematic review and meta-analysis of children with coronavirus disease 2019 (COVID-19). J Med Virol 2020;doi: 10.1002/jmv.26398. pmid: 32761898

10 Mithal LB, Machut KZ, Muller WJ, Kociolek LK. SARS-CoV-2 infection in infants less than 90 days old. J Pediatr 2020;224:150-2. doi: 10.1016/j.jpeds.2020.06.047 pmid: 32565095
11 Suwanwongse K, Shabarek N. Epidemiology, clinical features, and outcomes of hospitalized infants with COVID-19 in the Bronx, New York. Arch Pediatr 2020;27:400-1. doi: 10.1016/j.arcped.2020.07.009 pmid: 32891482

12 Wei M, Yuan J, Liu Y, Fu T, Yu X, Zhang ZJ. Novel coronavirus infection in hospitalized infants under 1 year of age in China. JAMA 2020;323:1313-4

doi: 10.1001/jama.2020.2131 pmid: 32058570

13 Dong Y, Mo X, Hu Y, etal. Epidemiology of COVID-19 among children in China. Pediatrics 2020;145:e20200702. doi: 10.1542/peds.2020-0702 pmid: 32179660

14 Poline J, Gaschignard J, Leblanc C, etal. Systematic SARS-CoV-2 screening at hospital admission in children:a French prospective multicenter study. Clin Infect Dis 2020;ciaa1044. doi: 10.1093/cid/ciaa1044. pmid: 32710743

15 Davies NG, Klepac P, Liu Y, Prem K, Jit M, Eggo RMCMMID COVID-19 working group. Age-dependent effects in the transmission and control of COVID-19 epidemics. Nat Med 2020;26:1205-11. doi: 10.1038/s41591-020-0962-9 pmid: 32546824

16 Centers for Disease Control and Prevention. CDC COVID Data Tracker. 2020. https://covid.cdc.gov/covid-data-tracker/\#datatracker-home

17 Mark EG, Golden WC, Gilmore M, etal. Community-onset severe acute respiratory syndrome coronavirus 2 infection in young infants: a systematic review. J Pediatr 2021;228:94-100.

18 Bixler D, Miller AD, Mattison CP, etalPediatric Mortality Investigation Team.

SARS-CoV-2-associated deaths among persons aged <21 years-United States, February 12-July 31, 2020. MMWR Morb Mortal Wkly Rep 2020;69:1324-9.

doi: 10.15585/mmwr.mm6937e4 pmid: 32941417

19 Leeb RT, Price S, Sliwa S, etal. COVID-19 trends among school-aged children -United States, March 1-September 19, 2020. MMWR Morb Mortal Wkly Rep 2020;69:1410-5. doi: 10.15585/mmwr.mm6939e2 pmid: 33001869

20 Song X, Delaney M, Shah RK, Campos JM, Wessel DL, DeBiasi RL. Comparison of clinical features of COVID-19 vs seasonal influenza A and B in US children. JAMA Netw Open 2020;3:e2020495. doi: 10.1001/jamanetworkopen.2020.20495 pmid: 32897374

21 Centers for Disease Control and Prevention. Health Department-reported cases of multisystem inflammatory syndrome in children (MIS-C) in the United States. https://www.cdc.gov/misc/cases/index.html

22 Feldstein LR, Rose EB, Horwitz SM, etalOvercoming COVID-19 InvestigatorsCDC COVID-19 Response Team. Multisystem inflammatory syndrome in US children and adolescents. N Engl) Med 2020;383:334-46. doi: 10.1056/NEJMoa2021680 pmid: 32598831

23 Godfred-Cato S, Bryant B, Leung J, etalCalifornia MIS-C Response Team. COVID-19-associated multisystem inflammatory syndrome in children-United States, March-July 2020. MMWR Morb Mortal Wkly Rep 2020;69:1074-80. doi: 10.15585/mmwr.mm6932e2 pmid: 32790663

24 Jiang L, Tang K, Levin M, etal. COVID-19 and multisystem inflammatory syndrome in children and adolescents. Lancet Infect Dis 2020;20:e276-88. doi: 10.1016/S1473-3099(20)30651-4 pmid: 32818434

25 Giovanni JE, Hrapcak S, Melgar M, Godfred-Cato S. Global reports of intussusception in infants with SARS-CoV-2 infection. Pediatr Infect Dis / 2021;40:e35-6. doi: 10.1097/INF.0000000000002946 pmid: 33105341

26 Dufort EM, Koumans EH, Chow E), etalNew York State and Centers for Disease Control and Prevention Multisystem Inflammatory Syndrome in Children Investigation Team. Multisystem inflammatory syndrome in children in New York State. N Engl J Med 2020;383:347-58. doi: 10.1056/NEJMoa2021756 pmid: 32598830

27 Diorio C, Henrickson SE, Vella LA, etal. Multisystem inflammatory syndrome in children and COVID-19 are distinct presentations of SARS-CoV-2. J Clin Invest 2020;130:5967-75. doi: 10.1172/JCl140970 pmid: 32730233

28 Consiglio CR, Cotugno N. CACTUS Study Team. The immunology of multisystem inflammatory syndrome in children with covid-19. Cell 2020;183:968-81.

29 Ouldali N, Toubiana J, Antona D, etal. Association of intravenous immunoglobulins plus methylprednisolone vs immunoglobulins alone with course of fever in multisystem inflammatory syndrome in children. JAMA 2021; doi: 10.1001/jama.2021.0694.

30 Park YJ, Choe YJ, Park O, etalCOVID-19 National Emergency Response Center, Epidemiology and Case Management Team. Contact tracing during coronavirus disease outbreak, South Korea, 2020. Emerg Infect Dis 2020;26:2465-8. doi: 10.3201/eid2610.201315 pmid: 32673193

31 Stein-Zamir C, Abramson N, Shoob H, etal. A large COVID-19 outbreak in a high school 10 days after schools' reopening, Israel, May 2020. Euro Surveill 2020;25:2001352. doi: 10.2807/1560-7917.ES.2020.25.29.2001352 pmid: 32720636

32 Schwartz NG, Moorman AC, Makaretz A, etal. Adolescent with COVID-19 as the source of an outbreak at a 3-week family gathering-four states, June-July 2020. MMWR Morb Mortal Wkly Rep 2020;69:1457-9. doi: 10.15585/mmwr.mm6940e2 pmid: 33031365

33 Szablewski CM, Chang KT, Brown MM, etal. SARS-CoV-2 transmission and infection among attendees of an overnight camp-Georgia, June 2020. MMWR Morb Mortal Wkly Rep 2020;69:1023-5. doi: 10.15585/mmwr.mm6931e1 pmid: 32759921

34 Zimmerman KO, Akinboyo IC, Brookhart MA, etalABC Science Collaborative. Incidence and secondary transmission of SARS-CoV-2 infections in schools. Pediatrics 2021e2020048090. doi: 10.1542/peds.2020-048090. pmid: 33419869

35 Hobbs CV, Martin LM, Kim SS, etalCDC COVID-19 Response Team. Factors associated with positive SARS-CoV-2 test results in outpatient health facilities and emergency departments among children and adolescents aged <18 years-Mississippi, September-November 2020. MMWR Morb Mortal Wkly Rep 2020;69:1925-9. doi: 10.15585/mmwr.mm6950e3 pmid: 33332298 
36 European Center for Disease Prevention and Control. Objectives for COVID-19 testing in school settings-first update, 21 August 2020. https://www.ecdc.europa.eu/sites/default/files/documents/covid-19-objectives-school-testing.pdf

37 Maryland Department of Health. COVID-19 guidance for child care facilities. 2021. https://earlychildhood.marylandpublicschools.org/system/files/filedepot/3/covid_guidance_full_080420.pdf

38 UK Department for Education. What to do if a child or adult is displaying symptoms, or is a confirmed case, of coronavirus (COVID-19) in an early years setting. 2021. https://assets.publishing.service.gov.uk/government/uploads/system/uploads/attachment_data/file/958657/What_to_do_if_a_child_or_adult_is_displaying_symptoms_or_is_a_confirmed_case_of_coronavirus_CoVl.pdf

39 Heald-Sargent T, Muller WJ, Zheng X, Rippe J, Patel AB, Kociolek LK. Age-related differences in nasopharyngeal severe acute respiratory syndrome coronavirus 2 (SARS-CoV-2) levels in patients with mild to moderate coronavirus disease 2019 (COVID-19). JAMA Pediatr 2020;174:902-3.

40 Centers for Disease Control and Prevention. Covid-19: Test for past infection. 2021. https://www.cdc.gov/coronavirus/2019-ncov/testing/serology-overview.html

41 Hatcher SM, Agnew-Brune C, Anderson M, etal. COVID-19 among American Indian and Alaska Native persons -23 states, January 31-July 3, 2020. MMWR Morb Mortal Wkly Rep 2020;69:1166-9. doi: 10.15585/mmwr.mm6934e1 pmid: 32853193

42 Swann OV, Holden KA, Turtle L, etallSARIC4C Investigators. Clinical characteristics of children and young people admitted to hospital with covid-19 in United Kingdom: prospective multicentre observational cohort study. BMJ2020;370:m3249. doi: 10.1136/bmj.m3249 pmid: 32960186

43 Horby P, Lim WS, Emberson JR, etalRECOVERY Collaborative Group. Dexamethasone in hospitalized patients with covid-19-preliminary report. N Engl J Med 2020;doi: 10.1056/NEJMoa2021436. pmid: 32678530

This article is made freely available for use in accordance with BMJ's website terms and conditions for the duration of the covid-19 pandemic or until otherwise determined by BMJ. You may use, download and print the article for any lawful, non-commercial purpose (including text and data mining) provided that all copyright notices and trade marks are retained. 